\title{
IPS: A new flexible framework for image processing
}

\author{
Otman ABDOUN \\ LaRIT, IbnTofail University \\ Faculty of Sciences, Kenitra, Morocco \\ Email: otman.fsk@gmail.com
}

\author{
Jaafar ABOUCHABAKA \\ LaRIT, IbnTofail University \\ Faculty of Sciences, Kenitra, Morocco \\ Email: abouch06-etudiant@yahoo.fr
}

\begin{abstract}
Image processing is a discipline which is of great importance in various real applications; it encompasses many methods and many treatments. Yet, this variety of methods and treatments, though desired, stands for a serious requirement from the users of image processing software; the mastery of every single programming language is not attainable for every user. To overcome this difficulty, it was perceived that the development of a tool for image processing will help to understanding the theoretical knowledge on the digital image. Thus, the idea of designing the software platform Image Processing Software (IPS) for applying a large number of treatments affecting different themes depending on the type of analysis envisaged, becomes imperative. This software has not come to substitute the existing software, but simply a contribution in the theoretical literature in the domain of Image Processing. It is implanted in the MATLAB platform: effective and simplified software specialized in image treatments in addition to the creation of Graphical User Interfaces (GUI) [5][6]. IPS is aimed to allow a quick illustration of the concepts introduced in the theoretical part. This developed software enables users to perform several operations. It allows the application of different types of noise on images, to filter images color and intensity, to detect edges of an image and to apply image thresholding by defining a variant threshold, to cite only a few.
\end{abstract}

Keywords-Image Processing; Noise; Filter; MATLAB; Edge detection.

\section{INTRODUCTION}

Image processing is a set of methods that can transform images or to extract information [4][6]. This is a very wide area, which is more and more applications:

- Much of the mail is now sorted automatically, thanks to the automatic recognition of the address,

- In the military field, devices capable of detecting and recognize automatically their targets,

- In industry, automatic control by vision is increasingly common in manufacturing lines,

- Image compression is experiencing a significant expansion in recent years, particularly through the development of Internet and digital television.

In this paper, we present an image processing software IPS, is developed in MATLAB, it represents a powerful tool for scientific calculations, and creating graphical user interfaces (GUI). The program is available for use with MATLAB or as a stand-alone application that can run on Windows and UNIX systems without requiring MATLAB and its toolboxes. The home interface of the IPS platform is shown in Fig.1

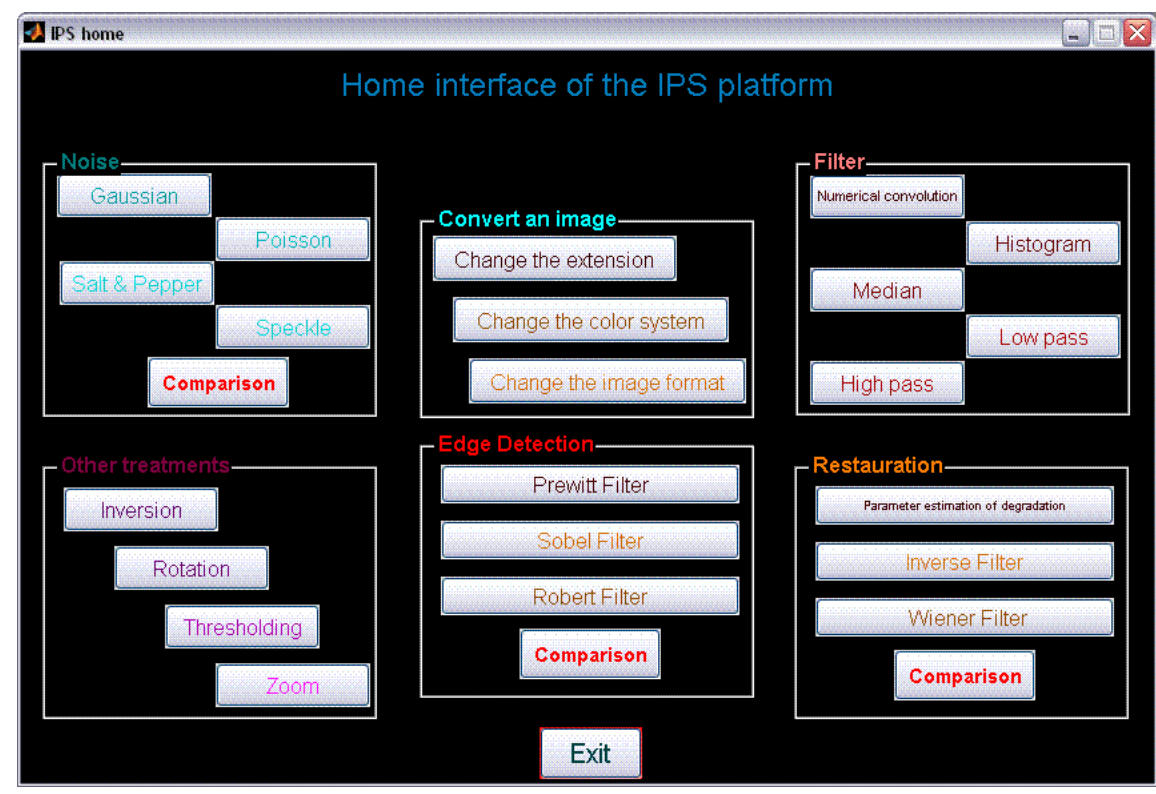

Figure. 1 : General overview of the program IPS 
The IPS platform is simple to use and easily scalable, and allows you to do the following:

- The application of different types of noise on images ;

- Equalize the histogram of an image blurred;

- Edge detection ;

- The filter color images and intensities;

- Thresholding of images by defining a threshold ranging;

- The change in format and extension images;

- Import and export images in various locations;

\section{HISTOGRAMME}

A histogram is a graph to represent the statistical distribution of pixel intensities of an image, that is to say the number of pixels for each intensity levels. By convention, a histogram represents the level of intensity in the $\mathrm{x}$-axis ranging from the darkest (left) to the lightest (right). In practice, for computing a histogram, it gives a number of quantization levels, and for each level, we count the number of pixels in the image corresponding to that level. MATLAB function that performs the calculation of a histogram is imhist [6]. It takes as parameters like the image name and the number of quantization levels desired.

\section{A. Histogram equalization}

The histogram equalization is a tool that is sometimes useful to enhance some images of poor quality (poor contrast, too dark or too bright, poor distribution of intensity levels, etc.) [4]. This is to determine a transformation of intensity levels that makes the histogram as flat as possible. If a pixel has intensity $\mathbf{i}$ in the original image, its intensity is smoothed image $\mathrm{f}(\mathrm{i})$. In general, we chose a step function, and determine the width and height of the various steps in order to flatten the image histogram equalized. MATLAB is performed by histogram equalization histeq $\mathrm{J}=(\mathrm{I}, \mathrm{n})$ where $\mathbf{I}$ denote the original image, $\mathbf{J}$ equalized image, and $\mathbf{n}$ is the number of intensity levels in the image equalized. Avoid choosing too large $n$ (for $n=64$ gives good results).
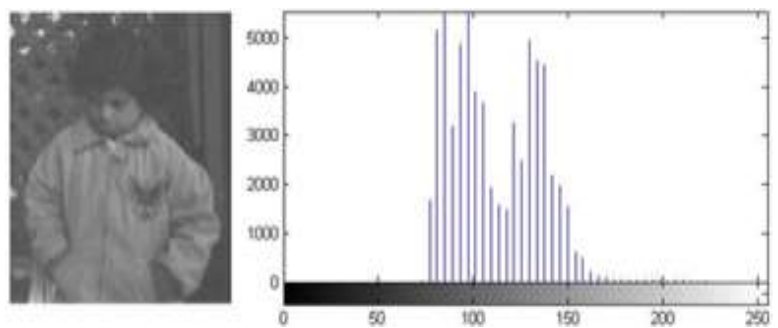

Figure 2.a : Original image blurred

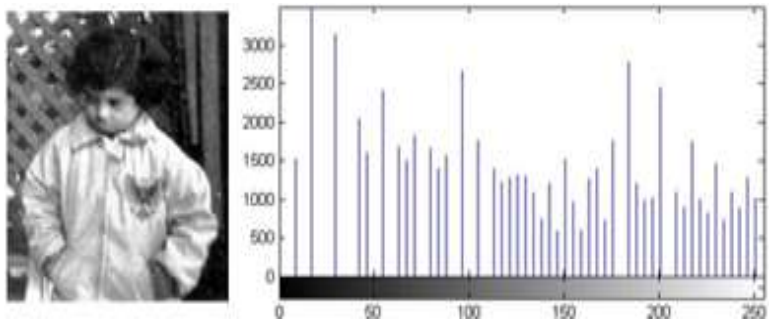

Figure 2.b. Equalized image

\section{B. Stretching the histogram}

The histogram stretching (also called "histogram linearization" or "expansion dynamics") is to allocate frequencies of occurrence of pixels on the width of the histogram. Thus it is an operation to modify the histogram so to the best allocation of intensities on the scale of values available. This amounts to extending the histogram so that the value of the lowest intensity is zero and the highest is the maximum value.

That way, if the values of the histogram are very close to each other, stretching will help to provide a better distribution to make even clearer light pixels and dark pixels close to the black.
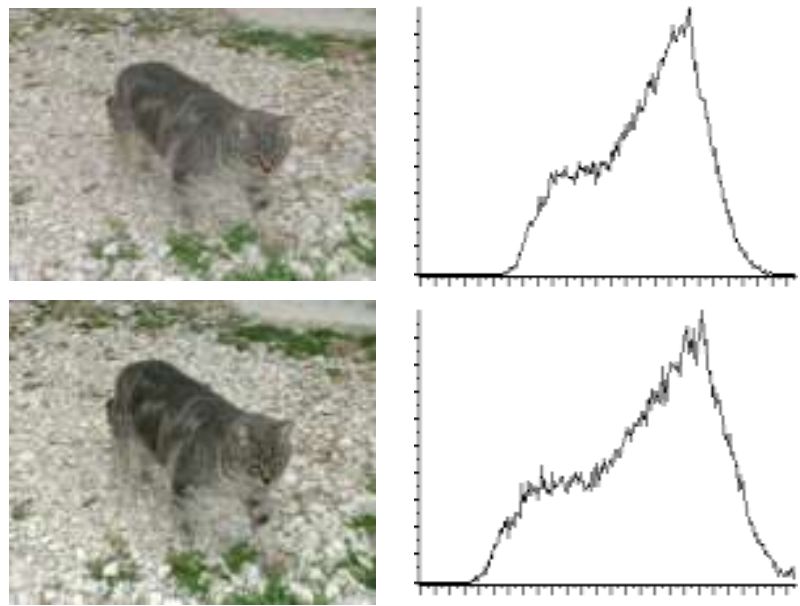

Figure 3. Stretching the histogram

It is thus possible to increase the contrast of an image. For example, a picture is too dark can become more "visible ".

\section{NOISE}

Characterizes the noise or interference noise signal, which is to say the parts locally distorted signal. Thus, the noise of an image means the image pixels whose intensity is very different from those of neighboring pixels.

Noise can come from various causes:

- Environment during the acquisition ;

- Quality of the sensor ;

- Quality of sampling.

There are several types of noise:

\section{A. Gaussian noise}

It's a sound, whose value is randomly generated following the Gaussian:

$$
B(x)=\frac{1}{\sqrt{2 \pi \sigma^{2}}} e^{\left(\frac{-(x-m)^{2}}{2 \sigma^{2}}\right)}
$$

With:

- $\sigma 2$ : Variance 
- $\mathrm{m}:$ median

\section{B. Speckle noise}

It is a noise (n), generated following the uniform law with an average of $0 \mathrm{~V}$ is a variance equal to 0.04 default. If $I$ is the original image, the noisy is defined by:

$$
\mathrm{J}=\mathrm{I}+\mathrm{n} * \mathrm{I}
$$

\section{Salt and Pepper noise}

This is a random signal generated by the Uniform Law. For the noise spectral density (D), it will be affected by noise $\mathrm{D}$ multiplied by the number of picture elements. Its principle is to :

- Determine the indices of its elements with a value less than half its density. Then assign 0 to the pixels corresponding to these indices in the image.

- Determine the indices of its elements with a value framed by half its density and its density. Then assign 1 to the pixels corresponding to the indices taken from the processed image

\section{POISSON noise}

It is an additive noise generated by the Poisson:

$$
P(x=k)=\frac{a^{k}}{k !} e^{-a}
$$

With a positive quantity is called the parameter of the law.

The function provided by MATLAB, which can generate noise that is IMNOISE, its syntax is:

\section{IMNOISE (I, TYPE)}

$\boldsymbol{I}:$ is the original image

TYPE: is the type of noise to apply, it may take the following values:

- 'GAUSSIAN': Gaussian noise to generate the function syntax IMNOISE will be as follows: IMNOISE (I, 'Gaussian', $\mathrm{m}, \mathrm{v}$ ), where $\mathrm{m}$ and $\mathrm{v}$ are respectively the mean and variance of the noise (Fig.4.a);

- 'POISSON': to generate the Poisson noise, the syntax is: IMNOISE(I,'Poisson') (Fig. 4.e);

- 'SALT \& PEPPER': to generate the noise with salt and pepper. The syntax is: IMNOISE(I,'salt \& pepper',D), where D is the density of noise (Fig.4.c);

- 'SPECKLE': to generate the speckle noise, the syntax is: IMNOISE(I,'speckle',V), where $\mathrm{V}$ is the noise variance (Fig.4.d).

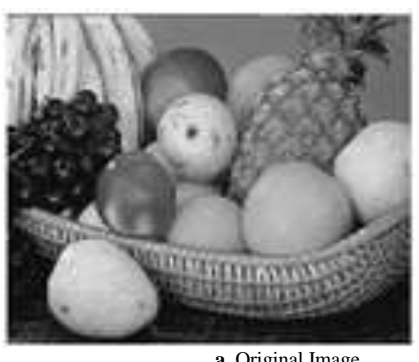

a. Original Image

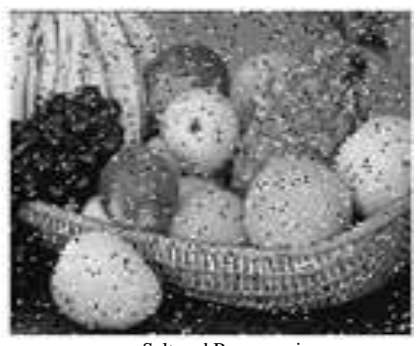

c. Salt and Pepper noise

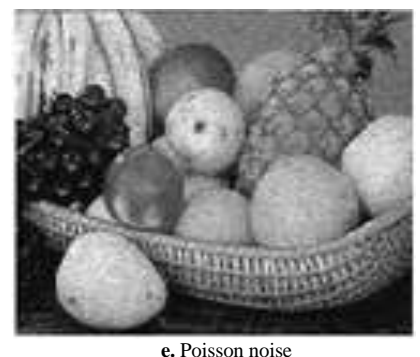

Figure 4. Noising image (a, b , c, d and e)

\section{IMAGE FILTERING}

A filter is a mathematical transformation (called convolution product) for, for each pixel of the area to which it applies, to change its value based on values of surrounding pixels, multiplied by coefficients.

The filter is represented by a table (matrix), characterized by its dimensions and its coefficients, whose center is the pixel concerned. The coefficients in Table determine the filter properties. An example of filter $3 \times 3$ is described in Fig 5:

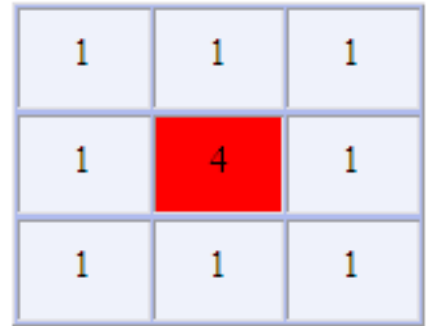

Figure 5. Filter $3 \times 3$

Thus the product matrix image, usually very large because it represents the initial image (array of pixels), the filter provides a matrix corresponding to the processed image.

We can say that filtering is to apply a transformation (called a filter) to all or part of a digital image by applying an operator. One generally distinguishes the following types of filters: 
- LOW-PASS filters, is to mitigate the components of the image having a high frequency (dark pixels). This type of filtering is generally used to reduce the image noise is why we usually talk about smoothing. The averaging filters are a type of low-pass filters whose principle is to average the values of neighboring pixels. The result of this filter is a fuzzy picture. MATLAB has a function to apply such filtering; it is the function Filter2 (Fig.6.c);

- Median filter is a nonlinear filter, which consists of replacing the gray level value at each pixel by the gray level surrounded by so many values that are higher than lower values in a neighborhood of the point considered. MATLAB has a function to apply such filtering; it is the function MEDFILT2 (Fig.6.e);

- HIGH-PASS FILTER, unlike the low-pass, reduce the low frequency components of the image and make it possible to accentuate the detail and contrast is why the term "filter accentuation "is sometimes used (Fig.6.d);

- Filters BANDPASS for obtaining the difference between the original image and that obtained by applying a low-pass filter.

- Directional Filters applying a transformation in a given direction.

Consider an image I and a two-dimensional filter h, filtering the image $\mathrm{I}$ by the filter $\mathrm{F}$ is an image whose luminance is given by:

$$
F(x, y)=\sum_{a, b} h(a, b) I(x+a, y+b)
$$
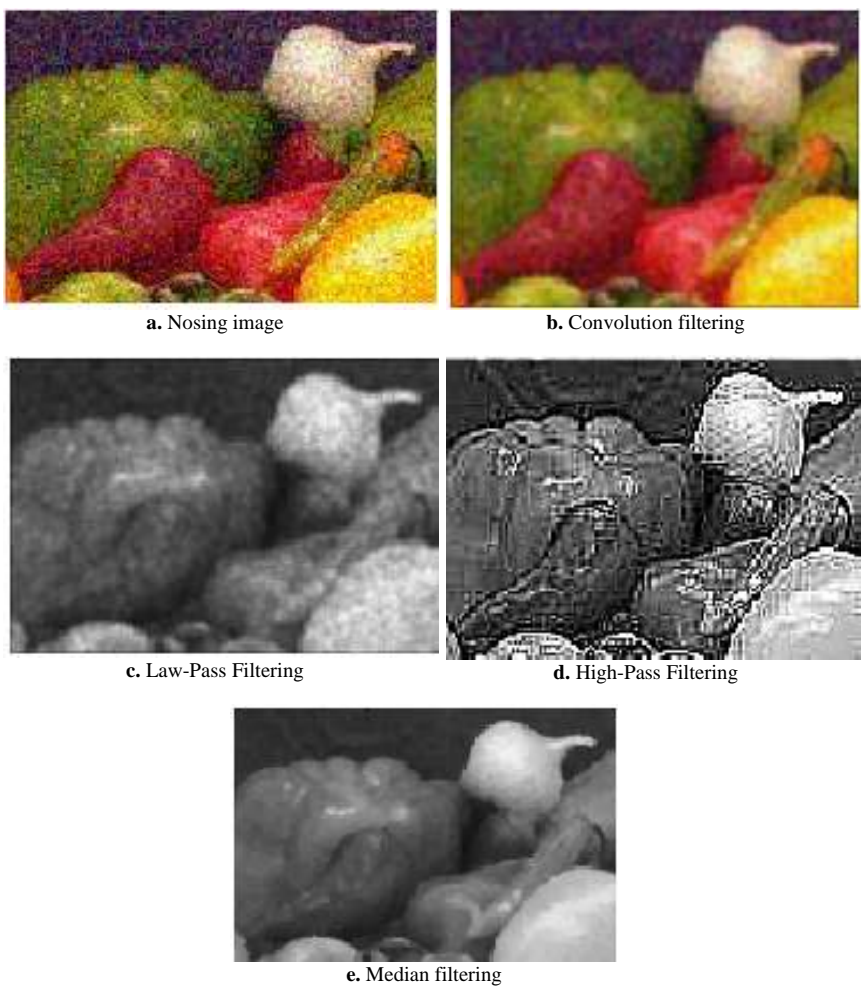

Figure 6. Image Filtering (a, b , c, d et e)

\section{RESTAURATION}

Restoring an image is to try to offset the damage suffered by this image [4][6]. The most common impairments are a blur or defocus shake. F image available is the result of degradation of the original image I. This degradation, when it's acts of defocus blur or camera shake, as a first approximation can be modeled by a linear filter $\mathrm{h}$, followed by the addition of noise $\mathrm{B}$ (Fig.7). The noise can account for the actual noise at the sensor and quantization noise from the digitization of the picture, but also and above all, the difference between the adopted model and reality. In general, we assume that it is a Gaussian white noise in the frequency domain.

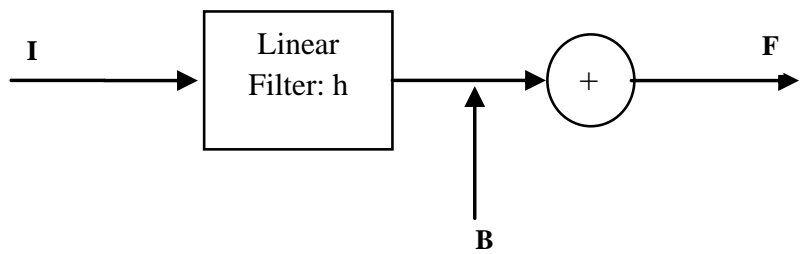

Figure 7. Principle of degradation

The restoration is calculated, from $\mathrm{F}$, an image $\mathrm{I}$ as close to the original image I [7]. To do this, we need to know the degradation. Degradation is sometimes assumed to be known, but in practice it is generally unknown, so we have estimation from the picture deteriorated, as shown in Fig.8:

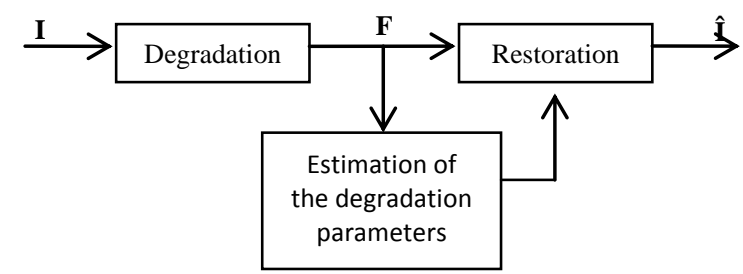

Figure 8. Principle of restoration

$$
\begin{gathered}
f(x, y)=\sum_{a, b} h(a, b) I(x+a \cdot y+b)+B(x, y) \\
=\left(h^{*} I\right)(x, y)+B(x, y)
\end{gathered}
$$

By taking the Fourier transform, assuming the images I and $\mathrm{F}$ periodic, we obtain:

$$
F(u, v)=H(u, v) I(u, v)+B(u, v)
$$

There are several types of degradation, mention the following:

\section{A. Defocus}

Each point on the scene then gives the picture a taskshaped disk; this task is much larger than the defocusing is important [6]. Degradation can be modeled by a linear filter $h$ whose coefficients $h(x, y) \lambda$ apply to the inside of discs and 0 outside (the value of $\lambda$ is calculated so that the sum is equal to 1 ).

To simplify the experiments, we assume below that the degradation is performed by a filter whose impulse 
response is a square (a square of $(2 \mathrm{~T}+1) *(2 \mathrm{~T}+1)$ pixels (where $\mathrm{T}$ is an integer). We then:

$a=\frac{1}{(2 T+1)^{2}}\left\{\begin{array}{l}h(x, y)=a \text { si }|x| \leq T \text { ou si }|\mathrm{y}| \leq T \\ h(x, y)=0 \text { sinon }\end{array}\right.$

(8)

The parameter to determine is $\mathrm{T}$.

\section{B. Candles}

If the deterioration is due to shake can be a first approximation, assuming that each point of the scene image gives a spot-shaped line segment (the orientation of this segment depends on the direction of move) [5]. This is modeled by a filter whose impulse response is in the shape of a segment.

For simplicity, assume here that this segment is horizontal. Thus, degradation is modeled by a horizontal filter

$$
\mathrm{h}=[\sqrt{\mathrm{a}}, \sqrt{\mathrm{a}}, \ldots ., \sqrt{\mathrm{a}}] \text { á } 2 T+1 \text { coefficients } .
$$

The value of coefficients is then:

$$
\sqrt{\mathrm{a}}=1 /(2 T+1)
$$

In reality, it is clear that the value of $\mathrm{T}$ is not necessarily right, that orientation may not be moved horizontally, the spot defocusing provides disk-shaped and not square shaped.

The degraded image is filtered by a filter $\mathrm{g}(\mathrm{x}, \mathrm{y})$ the inverse of $\mathrm{h}(\mathrm{x}, \mathrm{y})$. The problem is that the calculation of this filter is not always trivial, because it is the opposite in the sense of convolution. That's why we pass in the frequency domain using the Fourier transform. Indeed, this transform converts convolution into multiplication. In frequency, there will therefore:

$$
\mathrm{G}(\mathrm{u}, \mathrm{v})=\frac{1}{\mathrm{H}(\mathrm{u}, \mathrm{v})}
$$

In the previous sections we saw how to estimate $\mathrm{h}$ $(\mathrm{x}, \mathrm{y})$. Fourier transform, we deduce $\mathrm{H}(\mathrm{u}, \mathrm{v})$. We take care to observe the following precautions: Before applying the Fourier transform, fill with zeros h to reach the size of the image, otherwise the equation (10) contain extension functions different. Finally, the following equation gives us $\mathrm{G}(\mathrm{u}, \mathrm{v})$.

To restore the image, we calculate the spectrum of the restored image:

$$
\hat{\mathrm{I}}(\mathrm{u}, \mathrm{v})=\mathrm{G}(\mathrm{u}, \mathrm{v}) \mathrm{F}(\mathrm{u}, \mathrm{v})
$$

This involves applying the inverse filter in the frequency domain. Finally, an inverse Fourier transform applied to I ( $\mathrm{u}, \mathrm{v})$ gives the restored image $\mathrm{I}(\mathrm{x}, \mathrm{y})$.

To better understand the principle and limitations of this method, we will now express I (u, v) as a function of
I (u, v). Starting from the equation (11) and replacing $\mathrm{F}$ $(\mathrm{u}, \mathrm{v})$ by its expression (10), we obtain:

$$
\hat{\mathrm{I}}(\mathrm{u}, \mathrm{v})=\mathrm{G}(\mathrm{u}, \mathrm{v}) \mathrm{H}(\mathrm{u}, \mathrm{v}) \mathrm{I}(\mathrm{u}, \mathrm{v})+\mathrm{G}(\mathrm{u}, \mathrm{v}) \mathrm{B}(\mathrm{u}, \mathrm{v})
$$

Since, $G(u, v) H(u, v)=1$, on a :

$$
\hat{\mathrm{I}}(\mathrm{u}, \mathrm{v})=\mathrm{I}(\mathrm{u}, \mathrm{v})+\mathrm{G}(\mathrm{u}, \mathrm{v}) \mathrm{B}(\mathrm{u}, \mathrm{v})
$$

If the noise was zero, we would find exactly the original image. For nonzero noise, which will always be the case in practice, a problem arises when $\mathrm{H}(\mathrm{u}, \mathrm{v})$ becomes very low, because we will have a very high value of $\mathrm{G}(\mathrm{u}, \mathrm{v})$, which causes a strong noise amplification. A simple solution is to limit the possible values of $\mathrm{G}(\mathrm{u}, \mathrm{v})$ :

$$
\begin{aligned}
& \text { If } G(u, v)>S \text {, then } G(u, v)=S \\
& \text { If } G(u, v)<-S \text {, then } G(u, v)=-S
\end{aligned}
$$

Where, $\mathrm{S}$ is a positive threshold.

But because of this thresholding, $G$ is not exactly the inverse of $\mathrm{H}$, and degradation can't be totally eliminated.

\section{THRESHOLDING}

The operation called "simple thresholding" is set to zero all pixels with a gray level below a certain value (called threshold, English treshold) and the maximum value pixels with a higher value. Thus the result of thresholding is a binary image containing black and white pixels (Fig.9), is the reason why the term is sometimes used for binarization. Thresholding can highlight shapes or objects in an image. However the difficulty lies in choosing the threshold to adopt.

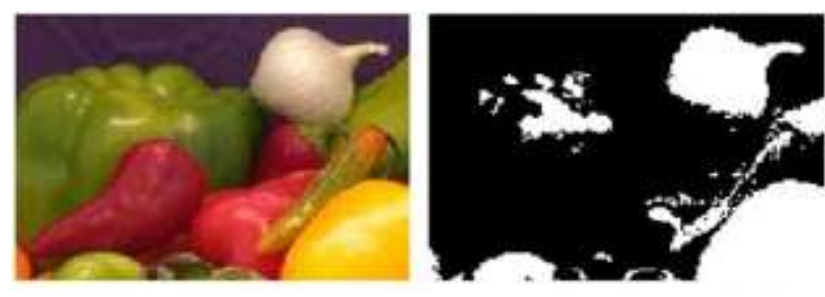

Figure 9. Image thresholding

VII. EDGE DETECTION

The contours are essential information for certain applications of image processing. In particular, the contours of an object can generally characterize its shape. Edge detection can be achieved with filters whose coefficients were carefully chosen. We discuss in this section, three sets of special filters: filters Prewitt, Roberts and Sobel.

A set of filters edge detection (Prewitt, Roberts and Sobel) consists of a pair of linear filters that detect the contours in two orthogonal directions: vertical and horizontal.

\section{A. Filter Prewitt}

The Prewitt filters horizontal and vertical are: 


$$
H=\left(\begin{array}{ccc}
1 & 1 & 1 \\
0 & 0 & 0 \\
-1 & -1 & -1
\end{array}\right) \quad V=\left(\begin{array}{ccc}
-1 & 0 & 1 \\
-1 & 0 & 1 \\
-1 & 0 & 1
\end{array}\right)
$$

From an image I, we calculate $\mathrm{Gh}$ and $\mathrm{Gv}$, image corresponding to the filtering of I h vs. we obtain the contour image single:

$$
G=\sqrt{G_{h}^{2}+G_{v}^{2}}
$$

We seek a binary contour image. For this, we must choose a threshold of detection. All pixels of $G$ whose intensity is above the threshold will increase to state 1 .

\section{B. Sobel Filtre}

The Sobel filters horizontal and vertical are:

$$
\mathrm{H}=\left(\begin{array}{ccc}
1 & 2 & 1 \\
0 & 0 & 0 \\
-1 & -1 & -1
\end{array}\right) \quad \mathrm{V}=\left(\begin{array}{ccc}
-1 & 0 & 1 \\
-2 & 0 & 2 \\
-1 & 0 & 1
\end{array}\right)
$$

\section{Robert Filtre}

The Robert filters horizontal and vertical are:

$$
\mathrm{H}=\left(\begin{array}{cc}
1 & 0 \\
0 & -1
\end{array}\right) \mathrm{V}=\left(\begin{array}{cc}
0 & 1 \\
-1 & 0
\end{array}\right)
$$
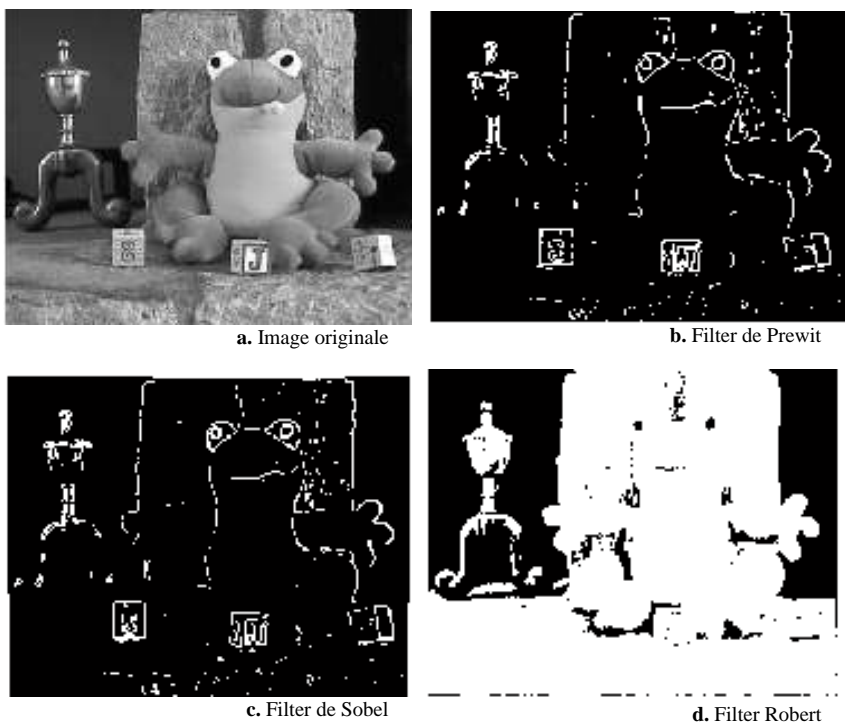

Figure 10. Edge detection of an image (a, b , c and d)

The choice of all of these treatments is justified by our desire to diversify the fields of applications enabled by our software IPS, which makes it very useful.

\section{CONCLUSION}

In this work, we developed software that we have appointed IPS and which is developed on MATLAB platform. This software is designed to allow the uninitiated to handle different programming features of MATLAB for image processing. IPS provides access to a set of operations such as: filtering, sound effects, restoration, edge detection and format change. It has a graphical interface easier to handle. We intend to improve this version by adding more functions.

\section{REFERENCES}

[1] Agarwal S., Awan A. and D. Roth, Learning to detect objects in images via a sparse, part-based representation, IEEE Trans. Pattern Anal. Mach. Intell. 26 (11) (2004), pp. 1475-1490

[2] Babaoğlu, Investigation of Reproduction Cells Using Digital Image Processing Techniques, Institute of Science, Selcuk Univerity, Konya (2004).

[3] Balasko B., J. Abonyi and B. Feil, Fuzzy Clustering and Data Analysis Toolbox for Use with MATLAB, Veszprem University, Hungary (2008)

[4] Blanchet G. et Charbit M. : Signaux et images sous MATLAB, Edition Hermes Sciences Europe Ltd, Paris 2001.

[5] Bres S., Jolion J.-M., LEbourgeois f. : Analyse et traitements des images numérique, Edition Lavoisier 2003.

[6] Burel G. : Introduction au traitement d'image simulation sous MATLAB, Edition Hermes Sciences Europe Ltd, Paris et Floch, Octobre 2001.

[7] Caroline CHAUX : Analyse en ondelettes M-bandes en arbre dual application à la restauration d'images, « http://igm.univ-mlv.fr/ LabInfo / rapportsInternes / 2007 / 03.pdf ».

[8] Chien S. and H. Mortensen, Automating image processing for scientific data analysis of a large image database, IEEE Trans. Pattern Anal. Mach. Intell. 18 (8) (1996), pp. 854-859.

[9] Eddins S.L., R.C. Gonzalez, and R.E. Woods, Digital image processing using MATLAB, Prentice-Hall, NJ (2004).

[10] Gonzalez, R.C., Woods, R.E., Digital Image Processing, third ed. Prentice-Hall Inc, Upper Saddle River, New Jersey, 2008.

[11] Nouvel, A., 2002. Description de concepts par un langage visuel pour un système d'aide à la conception d'applications de traitement d'images. Ph.D. Thesis, Paul Sabatier University, Toulouse, France, September.

[12] Russ, 2006 Russ, J.C., 2006. The Image Processing Handbook, fifth ed. (Image Processing Handbook), CRC Press Inc., Boca Raton, FL, USA. 